\title{
Synthesis of structural scheme of drive of adaptive multiple-link gripper
}

\author{
Aleksej Bogdanov ${ }^{1}$, Aleksandr Permyakov ${ }^{1}$ and Yulija Zhdanova ${ }^{2, *}$ \\ ${ }^{1}$ JSC 'SPA 'Android technics', Moscow, Russia \\ ${ }^{2}$ Institute of Cybernetics, Moscow Technological University (MIREA), Moscow, Russia
}

\begin{abstract}
The problem of construction of drive of multiple-link gripper capable to operate with human infrastructure objects is considered in the article. Technical specifications of structural scheme of actuating link group of a gripper are determined with reference to analysis of human hand finger bones motion. Structural scheme of system of motion transmission to the output links that realizes group drive and does not have kinematic correspondence of links is recommended. Synthesis of structural scheme is accomplished on the basis of providing of adaptability of output links location to the grasped object surface contour. The key point of the construction is variability of output link according to the interaction with grasped object. This is provided by passive power connections which are initiated between output links and motion transmission system links. Sequence of functioning of actuating link group is represented. Keywords: synthesis, structural scheme, drive, multiple-link gripper, actuating link group, passive power connections.
\end{abstract}

\section{Introduction}

Active works on designing of anthropomorphic robots (AR) are determined by factors of necessities and capabilities of designing of them as technical object capable to supply the place of human in performance of work (technological operations) in dangerous environment. Technical design specification of 'FEDOR' AR (as part of 'Spasatel' project financed by Advanced Research Foundation) covered 54 tests including wide range of activities specific to human: from going upstairs to using injection syringe. Technologies realized during AR designing are based on modern hardware components and original architecture of actuating complex and controlling system.

The whole range of the $\mathrm{AR}$ operations may be divided in several connected functions: analysis of outdoor environment, decision making, global moving in the outdoor environment, affecting the outdoor environment objects. Each function is essential and significant. At the same time the first two functions may be accomplished by operator in master-slave mode [1] and the third one may be accomplished by means of external subject.

Regional and local moving of gripper (AR working tool) and its interaction with outdoor environment objects are accomplished only by AR. Regional and local moving is provided by manipulator. Seven degrees of freedom manipulator makes it possible to remove the gripper to the localization point of required orientation angle and obstacle avoidance capability.

\section{Task description}

Analytical research of structural schemes of AR manipulators is accomplished considerably [2]. Analysis of gripping tool received far less consideration of researchers. On one side it is determined by a larger variety of potential constructional decisions, on the other side - by absence of universal methodology of its analysis. Interaction with undetermined objects of outdoor environment requires designing of cross functional gripper having both fine motor skills and power capacities. Multiple-link (anthropomorphic) gripper (MG) having kinematics similar to human hand [3] meets set requirements. MG has from three to five actuating link groups of the same structural scheme (Fig. 1, a). Each actuating link group (ALG) includes three links connected in sequence with each other by $B$ and $C$ rotating pairs with parallel axes (Fig. 1, b). Link 1 is connected with gripper base by two-degrees-of-freedom spherical pair $A$. Three from four degrees of freedom have parallel axes of rotation. Location of each $i$ link is determined by $\alpha_{i}$ relative rotation angle (Fig. 1, b). The total number of degrees of freedom is no less than 14.

Usage of two versions of drive schemes construction that provide links motion is possible. In this case drive shall mean complex of power section (electro-, pneumo-, hydraulic motor) including gear and motion transmission system (MTS) if necessary. According to the first version the drive is located on set in motion link or the previous one. In this case MTS may be absent and actuating group link that is set in motion is connected

\footnotetext{
Corresponding author: musrosjk@gmail.com
} 
with output link of power part directly. In the second version power part is placed on the manipulator link and the motion is transmitted through the MTS.

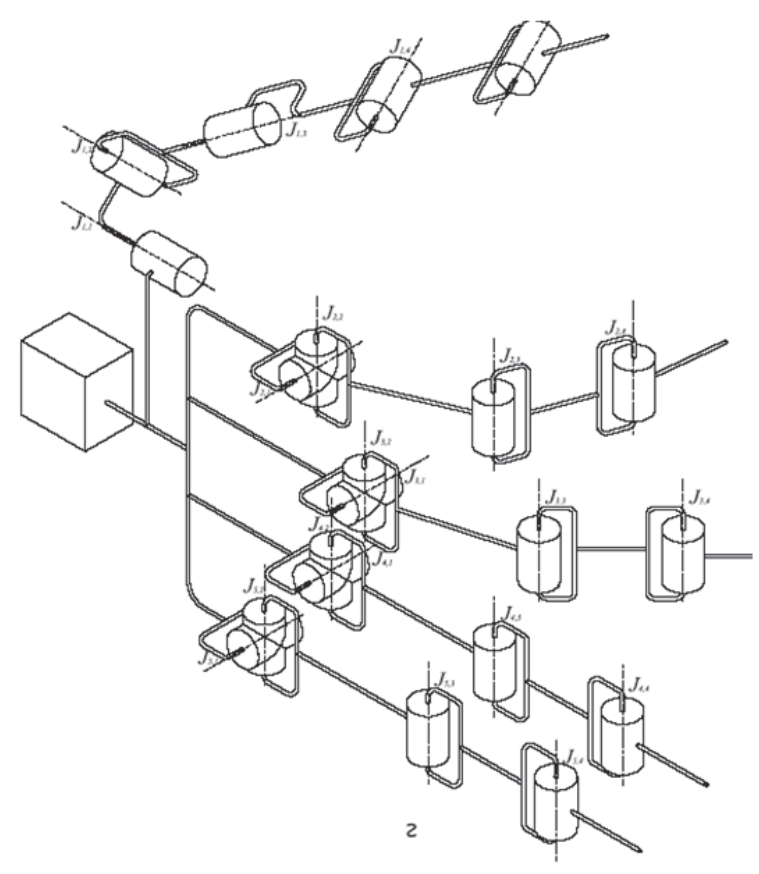

a



$\mathrm{b}$

Fig. 1. Structural schemes: a - anthropomorphic gripper, b actuating link group.

The first version is relatively easy in realization however it does not make it possible to use drives with sufficient force on the output link in virtue of restrictions placed on the links dimensions. The second version provides usage of high power motors. At the same time no more than 8-10 power parts may be placed on a manipulator link compactly. Usage of individual drive for each degree of freedom causes difficulties in its arrangement on a manipulator. The number of power parts used may be decreased by means of group drive. In this case the motion according to the degrees of freedom with parallel axes of rotation $A_{l}, B, C$ will be provided from one power part. The number of power parts for each AGL decreases from four to two. Kinematic correspondence of output links motion is typical for classic scheme of group drive construction. The employment of it in ALG results in stoppage of all output links as soon as one of them reaches the object surface.

\section{Formation of ALG drive terms of reference}

Kinematics of ALG must be similar to human hand kinematics and must provide interaction with external objects according to one of two scenarios either grasp or plucked grab. In the first case three phalanges operate with the object. This provides significant stress on the object surface and its safe fixation. In this case the location of each phalange is determined by proper generalized coordinate $\gamma_{i}$. In the second case only one distal phalange operates with the object.

Analysis of fingers grasp of different objects determined that distinct sequence is maintained. Table 1 represents values of relative rotation angles of phalanges (except thumb) while grasping cylinder object of $5 \mathrm{~mm}$ in diameter. Numerical values of 20-25 years old males with the hand size of 9 are noted. Interval in angles values is dictated predominantly by incongruous values between finger bones of different fingers and in a smaller degree by individual characteristics of examined men.

Table 1. Initial position and limiting position of phalanges.

\begin{tabular}{|cc|c|c|}
\hline \multirow{2}{*}{ Phalange } & \multicolumn{2}{c|}{ Phalange position, deg } \\
\cline { 3 - 4 } & & Initial $\gamma_{i .0}$ & Limiting $\gamma_{i \text {.Max }}$ \\
\hline Proximal & $\gamma_{1}$ & $0^{\circ}-20^{\circ}$ & $90^{\circ}-110^{\circ}$ \\
\hline Mesial & $\gamma_{2}$ & $0^{\circ}-35^{\circ}$ & $100^{\circ}-135^{\circ}$ \\
\hline Distal & $\gamma_{3}$ & $0^{\circ}-15^{\circ}$ & $45^{\circ}-75^{\circ}$ \\
\hline
\end{tabular}

Realizing of plucked grab is provided by means of motion of only just proximal phalange.

\section{Synthesis of structure of motion transmission system}

Analysis of phalanges motion sequence in the process of grasping objects of different surface contour makes it possible to formulate main aspects of finger functioning: - grasp is provided if only one generalized coordinate primary changes;

- absolute sequence of phalanges motion is maintained from proximal to distal;

- changing of generalized coordinate of each phalange is carried out until it reaches the object surface or the limiting value;

- each phalange has fixed value of limiting generalized coordinate.

\section{Technical specifications of drive structural scheme}

The results of the analysis make it possible to formulate technical specifications for development of ALG drive structure. 
- The motion of links should be realized from one power part of a drive.

- All the links are output with capability to change proper generalized coordinate independently.

- Limiting position of links must be adaptive (posses adaptive feature) to the grasped surface contour.

The last requirement leads to the fact that the order of changing of generalized coordinates and their final values are determined by the object surface contour. The motion of adaptive ALG may be provided from one or several motors.

MTS that meets the set technical specifications may be designed by realization of one or two standard approaches:

- usage of differential devices;

- usage of changing structural scheme.

Opportunity to employ the first approach is determined by the function of differential device capable to divide the motion of input link in several motions of output links.

The initial basis of the second approach includes the following statements:

- Structural scheme of MTS must provide motion of all output links of actuating group;

- While $i$ link is moving a part of structural scheme providing the transmission of motion to $i+1$ link must be blocked;

- The transmission of motion to $i$ link is provided in case of stoppage of $i+1$ link.

\section{Structural scheme of system of transmission of motion to the actuating group links}

The general structural scheme including the main scheme (Fig. 2, b) and MTS scheme must have three degrees of freedom; in such case two of them must be blocked initially. Its unblocking must happen in case of stoppage of one of output ALG links. As a result the general structural scheme has one degree of freedom at every instant. Realization of unit of blocking/unblocking of the kinematic pair degree of freedom is the most problematic issue of the approach. This problem may be solved by means of including of active or passive power connections.

The general structural scheme of ALG which has three output links (1,2 and 3) is represented in Figure 3. Link 4 is the input. Kinematic pair $A_{3}$ made of 4 and 1 links, and pair $A_{1}$ fit geometrically in a general structural scheme. Pair $B_{1}$ made of 8 and 2 links and pair $B$ have the same position similarly.

The number of degrees of freedom is determined by known formula

$$
W=3 n-2 p_{5}-p_{4} .
$$

Where $n$ is the number of moving links, $n=9$, $p_{5}$ is the number of kinematic pairs of the fifth type, $p_{5}=12$,

$p_{4}$ is the number of kinematic pairs of the fourth type, $p_{4}=0$.

$$
W=3 * 9-2 * 12=3 \text {. }
$$

Two passive power connections $a$ and $b$ by way of springs are included in the general structural scheme. Spring $a$ is fixed between links 4 and 1 , spring $b$ is fixed between links 8 and 2. If the links 1-3 do not contact with the object surface the connections $a$ and $b$ eliminate (block) relative motion in kinematic pairs $A_{3}$ and $B_{1}$. The number of kinematic pairs decreases to ten. In this case springs combine links 4,1 and 8,2 into one correspondingly. The number of links decreases to seven and the number of degrees of freedom is determined by correspondence (1) $W^{\mathrm{I}}=1$. The power part of the drive provides the motion of link 1 only from the initial position.
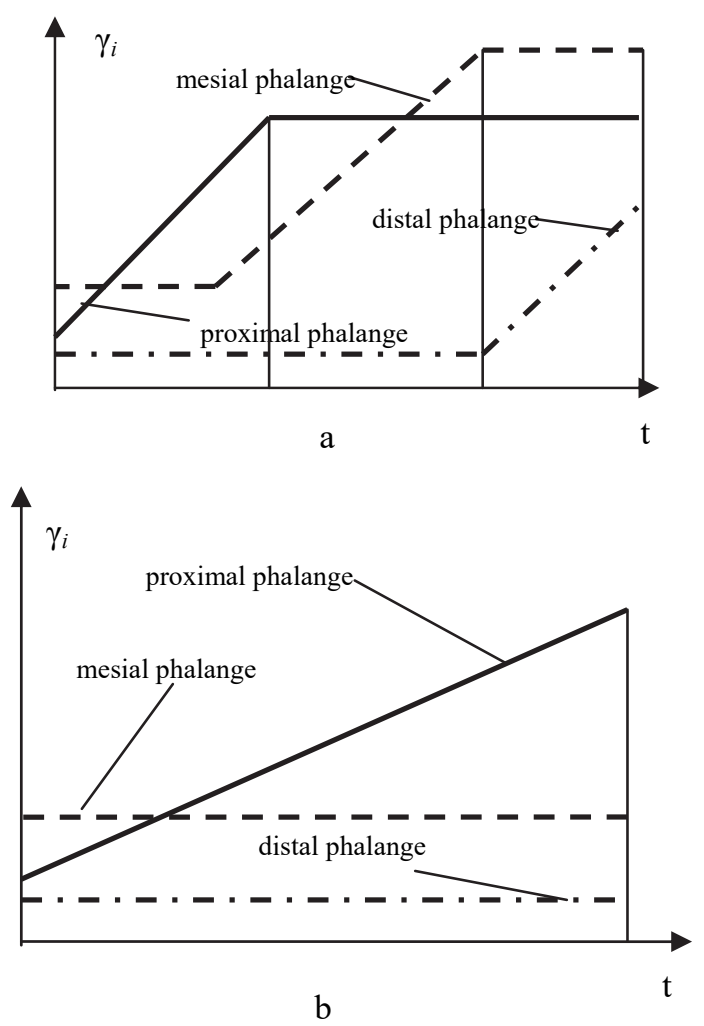

Fig. 2. Cyclogram of phalange motion: a - while grasping an object of minimum diameter $(\mathrm{d}=5 \mathrm{~mm}), \mathrm{b}$ - while plucked grabbing.



Fig. 3. The general scheme of ALG.

The process of grasping the object surface is carried out in three stages. The rotation of link 1 is realized from the initial position (Fig. 3). The generalized coordinate (Fig. 4, a) is changed. When link 1 reaches 
the object surface it stops (Fig. 4, b). Thus mobility of kinematic pair $A_{1}$ is not further realized. Generalized coordinate $\alpha_{1}$ takes the fixed value $\alpha_{1 \mathrm{~K}}$, the first stage of grasping is finished.

The stoppage of the link 1 and continuing motion of output link 4 provide unblocking of kinematic pair $A_{3}$. In this case spring $a$ is compressed. The number of mobile links is not changed as well as the number of kinematic pairs. Thus the number of degrees of freedom is $W^{\mathrm{II}}=W^{\mathrm{I}}$. The motion of input link 4 provides the rotation of link 2 in a kinematic pair $B$. The position of links 2 and 3 of the actuating group is determined only by generalized coordinate $\alpha_{2}$ (Fig. 4, c). The second stage of the object contour grasping is realized.
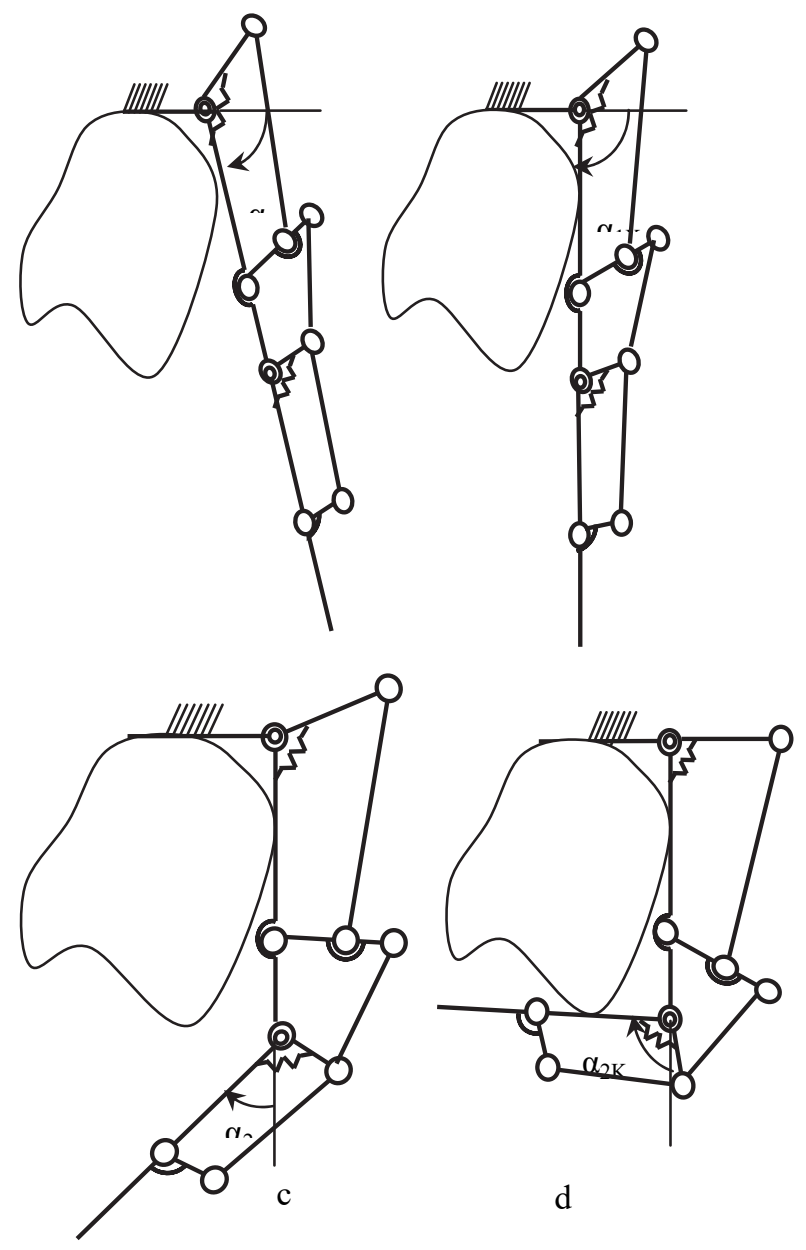

d

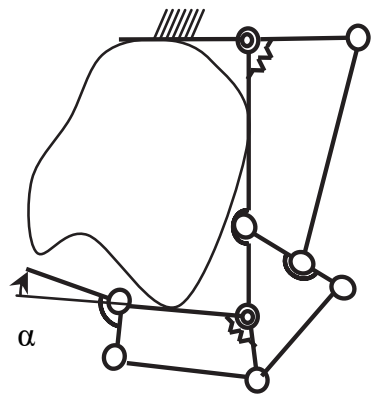

e



f
Fig. 4. Stages of the process of grasping the object contour by ALG links.
After the link 2 stops on the object surface (Fig. 4, d) the mobility in the kinematic pair $B$ is eliminated. Generalized coordinate $\alpha_{2}$ takes fixed value $\alpha_{2 \kappa}$. The second stage of the object contour grasping is finished.

The stoppage of link 2 provides unblocking of pair $B_{1}$. Link 8 has the opportunity to rotate then compression of spring $b$ (Fig. 4, e) is realized. The number of degrees of freedom stays the same $W^{\mathrm{III}}=W^{\mathrm{l}}$. The position of link 3 is determined by generalized coordinate $\alpha_{3}$. The third stage is finished when link 3 reaches the object contour (Fig. 4, f).

\section{Conclusion}

Group drive which structural scheme includes power connections should be used in design of grippers having kinematics similar to human hand at minimum number of motors. The location of them between links of actuating group and links of motion transmission system makes it possible to eliminate kinematic correspondence of motion. The found structural scheme of motion transmission system provides the grasp of the object contour by links of the gripper with adaptation of their position to the object shape.

\section{References}

1. A.A. Bogdanov, V.B. Sychkov, I.G. Zhydenko, I.M. Kutlubaev, Reshetnev Readings, 1 (16), 230-231 (2012)

2. I.G. Zhidenko, A.A. Bogdanov, I.M. Kutlubaev, V.B. Sychkov, Reshetnev Readings, 1, 278-280 (2013)

3. A.A Bogdanov, A.S. Gorbaneva, I.M. Kutlubaev, Yu.I. Kutlubaeva, Reshetnev Readings, 1, 273-274 (2014) 